\title{
Alteraciones cardíacas en los pacientes con enfermedad tiroidea
}

\section{Cardiac disorders in patients with thyroid disease}

\author{
Andrea N. Velandia-Rátiva ${ }^{1 *}$, Laura V. Zarta-Rengifo', Juan D. Guerrero-Peña1, \\ Karen T. Rangel-Castellanos ${ }^{1}$, Ledmar J. Vargas-Rodríguez ${ }^{1,2}$ y Edwar J. Rozo-Ortiz ${ }^{1,2}$ \\ ${ }^{1}$ Facultad de Ciencias de la Salud, Universidad de Boyacá; ${ }^{2}$ Departamento de Medicina Interna, Hospital San Rafael. Tunja, Colombia
}

\begin{abstract}
Resumen
Introducción: Al hablar de los trastornos funcionales de la glándula tiroides se hace referencia al aumento o la disminución en la producción de hormonas tiroideas; estos reciben el nombre de hipertiroidismo e hipotiroidismo, respectivamente. Son condiciones que han sido ampliamente estudiadas por el impacto negativo que tienen en el organismo, ya que ocasionan cambios en el metabolismo y generan alteraciones que llevan a que el paciente presente una serie de complicaciones graves, como son las enfermedades cardíacas. Objetivo: Identificar las alteraciones cardíacas en pacientes con enfermedad tiroidea. Método: Se realizó una revisión sistemática de las publicaciones disponibles en las bases de datos que hacen referencia a la enfermedad tiroidea y su relación con las alteraciones en el corazón. Resultados: En total se incluyeron 35 artículos publicados en los últimos 10 años, en distintos idiomas, en los que se evaluaba la asociación entre la enfermedad tiroidea y las alteraciones cardíacas. Conclusiones: Las enfermedades tiroideas están íntimamente relacionadas con cardiopatías que pueden empeorar la gravedad del cuadro clínico del paciente, e incluso asociarse con un incremento en la mortalidad de los mismos. Algunas de las cardiopatías mencionadas son cambios valvulares mixomatosos, disfunción endotelial, miocarditis, pericarditis aguda, vasoespasmo coronario, fibrilación atrial en el caso del hipertiroidismo, fibrosis endocárdica, aumento de la rigidez miocárdica, pericarditis, derrame pericárdico, estenosis coronaria y bradicardia sinusal por efecto del hipotiroidismo.
\end{abstract}

Palabras clave: Hipotiroidismo. Hipertiroidismo. Hormonas tiroideas. Cardiopatías. Insuficiencia cardíaca.

\section{Abstract}

Introduction: When speaking of functional disorders of the thyroid gland, reference is made to increased or decreased production of thyroid hormones. These were called hyperthyroidism and hypothyroidism respectively. They are pathologies that have been studied for the negative impact they have on the body, since they cause changes in the metabolism; thus generating alterations that lead to the patient presenting a series of serious complications, such as cardiac pathologies. Objective: To identify cardiac disorders in patients with thyroid disease. Method: A systematic review of the publications available in the databases that refer to thyroid pathology and its relationship with heart level disorders was performed. Results: $A$ total of 35 articles published in the last 10 years, in different languages, which evaluate the association between thyroid pathology and cardiac abnormalities are included. Conclusions: Thyroid pathologies are closely related to heart disease that can worsen

\section{Correspondencia:}

*Andrea N. Velandia-Rátiva

E-mail: anvelandia@uniboyaca.edu.co
Disponible en internet: 22-02-2022

Rev Colomb Cardiol. 2022;29(1):85-93

www.rccardiologia.com 0120-5633 / C 2021 Sociedad Colombiana de Cardiología y Cirugía Cardiovascular. Publicado por Permanyer. Este es un artículo open access bajo la licencia CC BY-NC-ND (http://creativecommons.org/licenses/by-nc-nd/4.0/). 
the severity of the patient's clinical condition, and may even be associated with an increase in their mortality. Some of the mentioned heart diseases are: myxomatous valve changes, endothelial dysfunction, myocarditis, acute pericarditis, coronary vasospasm, atrial fibrillation in the case of hyperthyroidism; endocardial fibrosis, increased myocardial rigidity, pericarditis, pericardial effusion, coronary stenosis, and sinus bradycardia due to hypothyroidism.

Keywords: Hypothyroidism. Hyperthyroidism. Thyroid Hormones. Heart disease. Heart failure.

\section{Introducción}

La prevalencia de enfermedad tiroidea en nuestro medio es alta. Esta condición patológica varía de acuerdo con factores como la edad, el sexo y la raza'. El hipertiroidismo es una anomalía tiroidea en la que se presentan niveles elevados de hormona tiroidea, debido a la secreción excesiva de la misma, o que sea de origen extratiroideo. En cambio, el hipotiroidismo es un trastorno en el que se producen bajos niveles de esta hormona, secundarios a una variación estructural o funcional ${ }^{2}$.

Estas son enfermedades endocrinas que pueden causar enfermedad cardiovascular, considerando la acción que ejercen las hormonas tiroideas sobre el corazón y los vasos sanguíneos, pues inducen cambios hemodinámicos y efectos sobre las células miocárdicas $^{3}$. También cabe señalar que el hipotiroidismo puede generar cambios, como disminución en la contractibilidad cardíaca, reducción del gasto cardíaco, disminución de la frecuencia cardíaca y disfunción diastólica ventricular; a diferencia del hipertiroidismo, en el que se encuentra mayor riesgo de desarrollar fibrilación auricular, infarto agudo de miocardio, embolia arterial y eventos cerebrovasculares ${ }^{4}$. Múltiples estudios observacionales muestran que la enfermedad tiroidea tiene efectos cardiovasculares ${ }^{4}$. El objetivo de esta investigación es identificar las alteraciones cardíacas en los pacientes con enfermedad tiroidea (hipertiroidismo o hipotiroidismo).

\section{Método}

Se realizó una revisión de la literatura científica de pacientes con hipertiroidismo e hipotiroidismo, en quienes se evaluó el desarrollo de alteraciones cardíacas y se identificó el grado de afectación cardíaca entendida como cambios del miocardio, el endocardio o el pericardio, cardiomegalia, infarto agudo de miocardio, arritmias o insuficiencia cardíaca congestiva, diagnosticados en base a los hallazgos clínicos y paraclínicos respectivos para cada una de las enfermedades.

Para ello se realizó una búsqueda de la literatura en las siguientes bases de datos: PubMed/Medline, ScienceDirect, Scopus, DOAJ, Embase, Cochrane, Direme,
Redalyc y SciELO. Para esto se utilizó la siguiente estrategia de búsqueda: (hypothyroidism OR hyperthyroidism OR Thyroids hormones) AND (heart diseases OR heart failure OR heart valve); y en idioma español: (hipotiroidismo $O$ hipertiroidismo $O$ hormonas tiroideas) Y (enfermedades del corazón O insuficiencia cardíaca $O$ válvula cardíaca).

Se incluyeron artículos e investigaciones que evaluaran uno o más de los desenlaces descritos, en tanto que se excluyeron investigaciones distintas a las mencionadas en los criterios de inclusión y artículos que no evaluaran los desenlaces descritos. Se limitó la búsqueda de la literatura a publicaciones de los últimos 10 años, en idiomas español e inglés.

\section{Resultados}

De acuerdo con los criterios establecidos y la estrategia de búsqueda, la exploración arrojó un total de 4476 artículos. Se excluyeron 3698 investigaciones tras la aplicación de los límites, quedando así 778 artículos. Posteriormente, se eliminaron 620 artículos por considerarlos no pertinentes para la resolución de la pregunta de investigación. Por último, se hizo la revisión de los artículos completos y se retiraron 123 por estar repetidos $(n=52)$ o no permitir su descarga $(n=71)$. De esta manera, se consideraron solo 35 estudios como pertinentes para dar respuesta a la pregunta de investigación. El proceso de selección de dichos estudios se muestra en el diagrama de flujo de la figura 1, de acuerdo con la declaración PRISMA. En total se incluyeron 35 artículos, cuyas características se muestran en la tabla 1.

\section{Tiroides y sistema cardiovascular}

La tiroides es una glándula que pesa aproximadamente 15-20 g en el adulto sano y está localizada en la parte inferior a la laringe y anterior a la tráquea. Está constituida por folículos cerrados que almacenan el coloide, sustancia conformada por la glucoproteína tiroglobulina, la cual contiene las hormonas T3 y T4. La función endocrina de estas hormonas es el incremento del metabolismo, pero cuando se produce ausencia de 


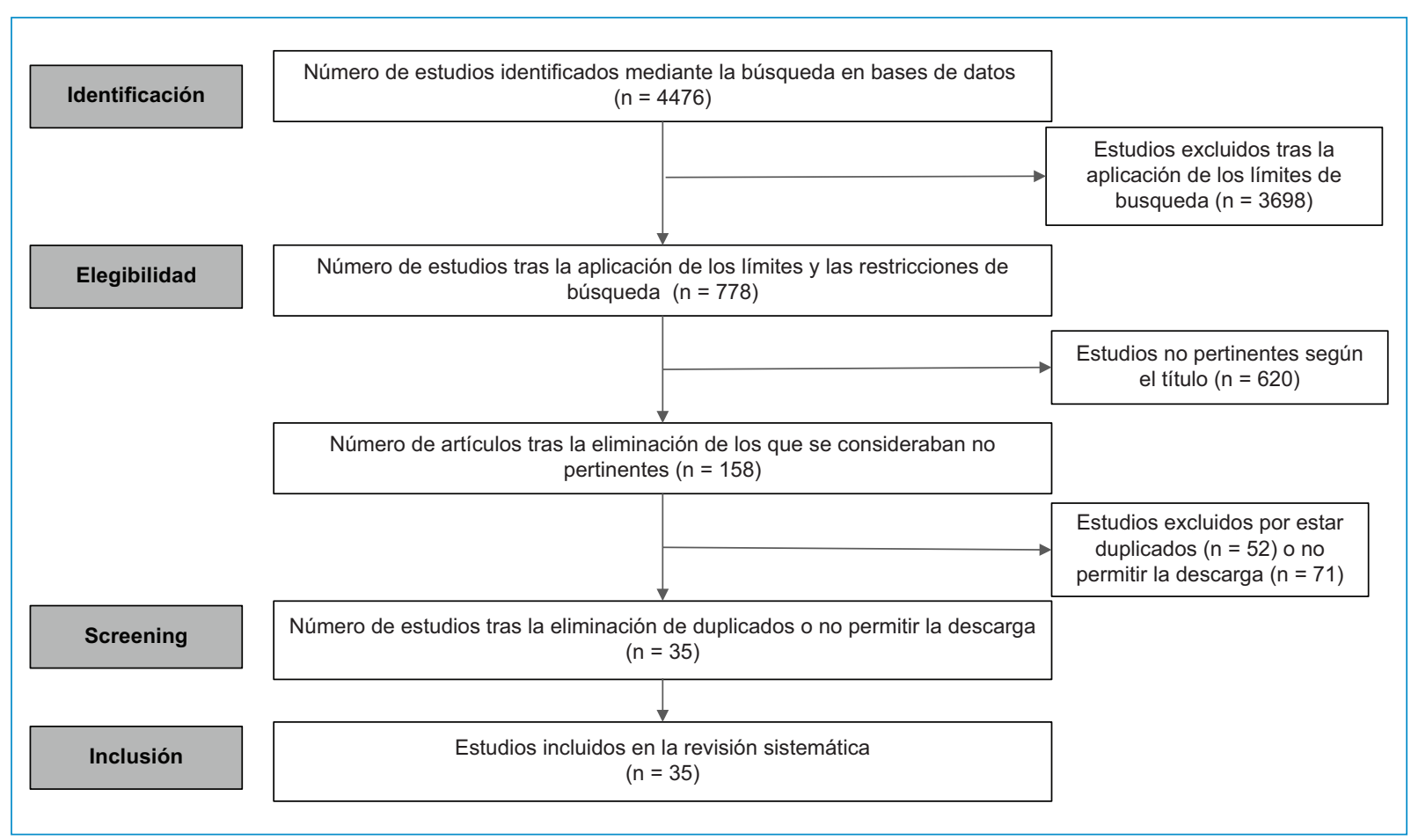

Figura 1. Proceso de selección de estudios de acuerdo con la declaración PRISMA.

estas (hipotiroidismo) se presenta un descenso en la tasa metabólica, a diferencia de cuando hay una cantidad excesiva de hormonas, que se incrementa la tasa metabólica ${ }^{5,6}$. Estas hormonas tienen acciones cardioprotectoras, especialmente a través de la acción de los receptores tiroideos $\alpha 1$ y $\beta 1$ de T3, donde actúan como antiapoptosis, antiinflamatorias, procontráctiles, antifibróticas, angiogénesis y regeneración, y tienen un efecto benéfico en cuanto al micro- $\operatorname{ARN}^{7,8}$.

La disfunción tiroidea es un motivo frecuente de consulta. Esta puede afectar al $15 \%$ de las mujeres y a un porcentaje menor de los hombres. Asimismo, es importante identificar los signos y síntomas cardiovasculares, como son, en el hipertiroidismo, la taquicardia, la fibrilación auricular y la hipertensión sistólica, entre otros, y los efectos opuestos generados en el hipotiroidismo ${ }^{9}$.

\section{Hipotiroidismo}

Entre un $4 \%$ y un $10 \%$ de la población cursa con hipotiroidismo $^{10}$. La característica principal es la disminución de T4 y T3, con un ascenso en los niveles de la hormona estimulante de la tiroides (TSH); este es un mecanismo compensatorio ante los valores bajos de estas hormonas ${ }^{6,11}$. Las manifestaciones clínicas clásicas de esta son fatiga, lentitud, voz ronca, estreñimiento, reflejo tendinoso distal retardado y cambios tanto en la piel como cardiovasculares ${ }^{11}$. El hipotiroidismo se asocia con baja disponibilidad del óxido nítrico endotelial, además de relajación alterada del músculo liso vascular, lo que conlleva disminución del gasto cardíaco y, como consecuencia, genera un aumento en la rigidez arterial, incrementando así la resistencia vascular sistémica. A nivel molecular, estas alteraciones resultan de la expresión reducida del retículo sarcoplásmico $\mathrm{Ca}^{2+}$-ATPasa y el aumento de la expresión de fosfolambán, que inhibe la ATPasa. Las hormonas tiroideas también afectan el sistema renina-angiotensina-aldosterona (los sustratos de renina se sintetizan en el hígado bajo el estímulo de T3). Por lo tanto, en un estado hipotiroideo, la presión arterial diastólica aumenta, la presión del pulso se estrecha y los niveles de renina disminuyen. Esto resulta en hipertensión diastólica que, a menudo, es sensible al sodio ${ }^{10}$.

\section{Alteraciones cardíacas en pacientes con HIPOTIROIDISMO}

\section{Alteración de las válvulas cardíacas}

Es poco común, pero algunos pacientes con hipotiroidismo crónico pueden presentar cambios valvulares 
Rev Colomb Cardiol. 2022;29(1)

Tabla 1. Características de los artículos incluidos en la revisión

\begin{tabular}{|c|c|c|c|}
\hline Autor(es) & Nombre del artículo & Año & Idioma \\
\hline Chaves $^{1}$ & $\begin{array}{l}\text { Prevalencia de la disfunción tiroidea en la población adulta mayor de la consulta } \\
\text { externa del hospital de San José }\end{array}$ & 2018 & Español \\
\hline Kumar et al. ${ }^{2}$ & Patología estructural y funcional & 2015 & Español \\
\hline Soto y Verbeke ${ }^{3}$ & Disfunción tiroidea y corazón & 2015 & Español \\
\hline Espinosa $^{4}$ & Mujer, corazón y tiroides & 2017 & Español \\
\hline Vélez et al. $^{5}$ & $\begin{array}{l}\text { La glándula tiroides en el paciente críticamente enfermo: abordaje fisiológico y } \\
\text { revisión de la literatura }\end{array}$ & 2019 & Español \\
\hline Dischinger y Fassnacht ${ }^{6}$ & $\begin{array}{l}\text { Thyroid gland and the heart: pathophysiological background, diagnostic and } \\
\text { therapeutic consequences }\end{array}$ & 2018 & Alemán \\
\hline Martínez $^{7}$ & Thyroid hormones and heart failure & 2016 & Inglés \\
\hline $\operatorname{Dan}^{8}$ & Thyroid hormones and the heart & 2016 & Inglés \\
\hline Danzi y Klein ${ }^{9}$ & Thyroid abnormalities in heart failure & 2020 & Inglés \\
\hline Udovcic et al. ${ }^{10}$ & Hypothyroidism and the heart & 2017 & Inglés \\
\hline Grais y Sowers ${ }^{11}$ & Thyroid and the heart & 2014 & Inglés \\
\hline Martínez et al. ${ }^{12}$ & $\begin{array}{l}\text { Subclinical hypothyroidism might increase the risk of postoperative atrial fibrillation } \\
\text { after aortic valve replacement }\end{array}$ & 2015 & Inglés \\
\hline Iervasi y Nicolini ${ }^{13}$ & $\begin{array}{l}\text { Thyroid hormone and cardiovascular system: from basic concepts to clinical } \\
\text { application }\end{array}$ & 2013 & Inglés \\
\hline Klein y Danzi ${ }^{14}$ & Thyroid disease and the heart & 2016 & Inglés \\
\hline Jabbar et al..$^{15}$ & Thyroid hormones and cardiovascular disease & 2017 & Inglés \\
\hline Vargas y Bonelo ${ }^{16}$ & Thyroid dysfunction and heart failure: mechanisms and associations & 2017 & Inglés \\
\hline Biondi y Klein ${ }^{17}$ & Hypothyroidism as a risk factor for cardiovascular disease & 2004 & Inglés \\
\hline Vargas et al. ${ }^{18}$ & Effects of thyroid hormones on the heart & 2014 & Inglés \\
\hline Mirwais et al. ${ }^{19}$ & Hypothyroidism causing pericardial effusion: a case report & 2019 & Inglés \\
\hline Delitala et al. ${ }^{20}$ & Subclinical hypothyroidism and cardiovascular risk factors & 2019 & Inglés \\
\hline Badawy et al. ${ }^{21}$ & Thyroid stimulating hormone as risk factor for coronary heart disease & 2013 & Inglés \\
\hline Reddy et al. ${ }^{22}$ & Atrial fibrillation and hyperthyroidism: a literature review & 2017 & Inglés \\
\hline Khan et al. ${ }^{23}$ & $\begin{array}{l}\text { Thyroid and cardiovascular disease: a focused review on the impact of } \\
\text { hyperthyroidism in heart failure }\end{array}$ & 2020 & Inglés \\
\hline Pierre et al. ${ }^{24}$ & Thyrotoxic valvulopathy: case report and review of the literature & 2017 & Inglés \\
\hline Kobayashi et al. ${ }^{25}$ & Unusual manifestation of Graves' disease: ventricular fibrillation & 2015 & Inglés \\
\hline Gussak y Antzelevitch ${ }^{26}$ & $\begin{array}{l}\text { Early repolarization syndrome: clinical characteristics and possible cellular and } \\
\text { ionic mechanisms }\end{array}$ & 2000 & Inglés \\
\hline Ueno et al. ${ }^{27}$ & $\begin{array}{l}\text { Ventricular fibrillation associated with early repolarization in a patient with thyroid } \\
\text { storm }\end{array}$ & 2010 & Inglés \\
\hline Mavrogeni et al..$^{28}$ & $\begin{array}{l}\text { Hyperthyroidism induced autoimmune myocarditis. Evaluation by cardiovascular } \\
\text { magnetic resonance and endomyocardial biopsy }\end{array}$ & 2012 & Inglés \\
\hline Lancaster et al. ${ }^{29}$ & $\begin{array}{l}\text { Acute autoimmune myocarditis as a manifestation of Graves' disease: a case } \\
\text { report and review of the literature }\end{array}$ & 2019 & Inglés \\
\hline Alam y Zaman ${ }^{30}$ & Case study of thyrotoxic cardiomyopathy & 2019 & Inglés \\
\hline Smith y Hegedüs ${ }^{31}$ & Graves' disease & 2016 & Inglés \\
\hline
\end{tabular}


Tabla 1. Características de los artículos incluidos en la revisión (Continuación)

\begin{tabular}{|l|l|l|l|}
\hline Autor(es) & Nombre del estudio & Año & Idioma \\
\hline Koo et al. $^{32}$ & Acute recurrent pericarditis accompanied by Graves' disease & 2012 & Inglés \\
\hline Mirić et al. ${ }^{33}$ & Thyrotoxicosis as the cause of acute recurrent perimyocarditis & 2018 & Inglés \\
\hline Razvi et al. ${ }^{34}$ & Thyroid hormones and cardiovascular function and diseases & 2018 & Inglés \\
\hline Mejia et al. ${ }^{35}$ & Hyperthyroidism and the heart & 2017 & Inglés \\
\hline
\end{tabular}

mixomatosos ${ }^{11}$. En el hipotiroidismo subclínico se requiere que los niveles de T4 libre estén dentro del rango normal, pero que los niveles séricos de TSH estén moderadamente elevados ${ }^{12}$. En un estudio se evaluaron 467 pacientes para determinar la relación existente entre el hipotiroidismo subclínico y el desarrollo de fibrilación auricular en el posoperatorio inmediato de cirugía de reemplazo de válvula aórtica con circulación extracorpórea. En este se demostró que el hipotiroidismo subclínico se comporta como un factor de riesgo para desarrollar fibrilación auricular posoperatoria en pacientes sometidos a reemplazo valvular aórtico con circulación extracorpórea, puesto que la incidencia fue del $57 \%$ en el grupo con hipotiroidismo subclínico frente al $30.3 \%$ en el grupo sin hipotiroidismo. Sin embargo, en este estudio se recalca que se necesitan más estudios para determinar si la terapia de reemplazo de T4 preoperatoria y la profilaxis de fibrilación auricular más agresiva previenen este tipo de complicación en pacientes sometidos a reemplazo de válvula aórtica ${ }^{12}$. Asimismo, se debe tener en cuenta el pequeño número de pacientes con hipotiroidismo subclínico, puesto que de los 467 pacientes estudiados solo 35 eran casos de hipotiroidismo subclínico, lo que se considera una limitante en dicho estudio, además de la falta de medición de los niveles de T3 y T3 libre. Esto es un impedimento para confirmar el "síndrome de T3 bajo" en los pacientes con hipotiroidismo subclínico después de la operación ${ }^{12}$.

\section{Alteración del endocardio}

Rara vez se presentan fibrosis endocárdica, miocardiopatía o cambios valvulares mixedematosos ${ }^{11}$.

\section{Alteración del miocardio}

El hipotiroidismo, como la hipertensión y los efectos del envejecimiento, conducen a una menor actividad de SERCA2 y, por tanto, a un deterioro del ciclo del calcio, lo que resulta en un aumento de la rigidez miocárdica y del consumo de $\mathrm{O}_{2}{ }^{13}$, y en disfunción diastólica ventricular izquierda. Incluso, el hipotiroidismo subclínico puede aumentar la incidencia de insuficiencia cardíaca y disfunción diastólica ${ }^{14-16}$. La triyodotironina estimula la transcripción de SERCA2 mientras reprime simultáneamente la expresión de fosfolambán. La última proteína ejerce un efecto inhibitorio sobre el ciclo del calcio mediado por SERCA2 y, por lo tanto, el efecto de la hormona tiroidea en estas dos proteínas es sinérgico. La tasa de liberación de calcio y su recaptación en el retículo sarcoplasmático son determinantes importantes de la contractilidad sistólica y la relajación diastólica. Por consiguiente, la regulación de las cantidades de estas proteínas en el miocito explica los efectos observados de T3 en la contractilidad miocárdica ${ }^{6,13,17}$. Los pacientes con hipotiroidismo subclínico presentan disminución en los niveles de T3, lo que genera una relación importante con la fibrilación posoperatoria tras un reemplazo valvular aórtico con circulación extracorpórea ${ }^{12}$. Esto se debe a una disminución de la actividad de la adenosina trifosfatasa del retículo sarcoplasmático y una reducción de las corrientes de $\mathrm{Ca}^{2+}$ en los miocitos cardíacos, observado en la remodelación iónica arritmogénica ${ }^{12}$.

\section{Alteración del pericardio}

Aproximadamente un 4\% de los pacientes desarroIlan pericarditis ${ }^{11,15,18}$, e incluso algunos pacientes con hipotiroidismo grave llegan a desarrollar derrame pericárdico en un 3-6\% de los $\operatorname{casos}^{15}$, cuya causa no es muy clara, si bien se cree que se debe a la retención de volumen que hay en estos pacientes. Existe una extravasación en la que sale líquido rico en proteínas a la superficie del corazón, por un aumento en la permeabilidad del capilar ${ }^{11,15}$; en algunos casos, al examen físico el líquido se observa de coloración amarillenta, debido a la presencia de cristales de colesterol que le otorgan esta tonalidad ${ }^{11}$. Rara vez se 
produce taponamiento pericárdico ${ }^{15}$, sobre todo en sujetos en quienes predominan niveles bajos de $\mathrm{T} 4^{18}$. Recientemente se presentó un reporte de caso de un varón de 68 años con un historial médico extenso de consumo de alcohol y tabaco, incumplimiento de la medicación, enfermedad de Graves tratada con ablación con yodo radiactivo e hipotiroidismo resultante, enfermedad pulmonar obstructiva crónica, hipertensión, trombosis venosa profunda con colocación de filtro de vena cava inferior y trastorno bipolar, presentado en la sala de emergencias por tos e intoxicación con alcohol. La radiografía de tórax mostró derrame pericárdico, que se confirmó con tomografía computarizada de tórax y ecocardiografía. El nivel de TSH fue de $31.42 \mu \mathrm{UI} / \mathrm{ml}$ (rango normal: 0,4-5,5 $\mu \mathrm{UI} / \mathrm{ml}$ ), con T4 libre indetectable y antecedentes de enfermedad tiroidea, apuntando hacia un hipotiroidismo incontrolado como causa probable de su derrame pericárdico ${ }^{19}$.

\section{Alteración en la circulación coronaria}

La disminución de la hormona tiroidea actúa directamente sobre el corazón y la vasculatura, e influye de manera indirecta en la hemodinámica cardiovascular, lo que conduce a una disminución en la contractibilidad cardíaca, pero a su vez a aumentar la resistencia vascular y a causar estenosis coronaria ${ }^{6,9}$. De igual manera, en el hipotiroidismo subclínico se ha observado el posible vínculo con la enfermedad coronaria, en especial cuando hay niveles altos de TSH y niveles significativamente elevados en adultos con TSH de $10 \mathrm{mUI} / \mathrm{l}$ o más. El principal factor es el aumento en los niveles de lípidos en suero, así como rigidez arterial, disfunción endotelial, homocisteína, niveles elevados de proteína $\mathrm{C}$ reactiva, parámetros de coagulación alterados e hipertensión diastólica ${ }^{20,21}$.

\section{Alteración del sistema eléctrico del corazón}

Los cambios más comunes que aparecen son bradicardia sinusal por la disfunción del nodo sinusal y el fallo en la capacidad de este para aumentar la frecuencia cardíaca en situaciones de estrés; otros pueden presentar taquicardia, la cual puede estar acompañada de taquicardia helicoidal, bloqueo auriculoventricular y prolongación del $\mathrm{QT}^{11,18}$.

\section{Hipertiroidismo}

El hipertiroidismo o tirotoxicosis se debe a la liberación excesiva de hormona tiroidea secundaria a una glándula tiroides hiperactiva o a la liberación pasiva de la hormona almacenada. También puede producirse por el tratamiento excesivo con hormona tiroidea. Generalmente, se considera abierto o subclínico, dependiendo de la gravedad bioquímica ${ }^{22}$. El hipertiroidismo manifiesto se define como una TSH suprimida y valores elevados de T3 o T4 libre estimada. El hipertiroidismo subclínico cursa con niveles de TSH séricos bajos o indetectables y valores dentro del rango de referencia normal para T3 y T4 libre. El hipertiroidismo debe considerarse la enfermedad potencial siempre que el nivel de TSH sea subnormal ${ }^{22}$. La insuficiencia cardíaca representa la presentación clínica inicial en aproximadamente el $6 \%$ de los pacientes con hipertiroidismo, y la mitad tienen disfunción ventricular izquierda ${ }^{13,15,16,23}$.

\section{Alteraciones cardíacas en pacientes con HIPERTIROIDISMO}

\section{Alteración de las válvulas cardíacas}

En el artículo Thyrotoxic valvulopathy: case report and review of the literature se comenta el caso de una paciente que presentó una válvula tricúspide mixedematosa con regurgitación tricuspídea ${ }^{24}$. En el mismo se comenta sobre reportes de caso similares, que se han presentado en pacientes con alguna valvulopatía, la cual ha sido resuelta luego del tratamiento adecuado para el hipertiroidismo ${ }^{24}$. Se cree que la regurgitación tricuspídea está causada por la dilatación del ventrículo derecho secundaria al aumento del retorno venoso. Sin embargo, en el artículo mencionado, la paciente cursó con una válvula tricúspide mixomatosa, con una regurgitación tricúspidea importante que pudo ser causada por una combinación de regurgitación tricuspídea primaria debida a la degeneración mixomatosa de las valvas, además de un componente secundario de regurgitación tricuspídea relacionado con la dilatación del ventrículo derecho. Esto se fundamenta en que la degeneración mixomatosa se caracteriza por una apariencia desordenada de haces de colágeno, que finalmente conducen a un tejido que se estira y se rompe con mayor facilidad. Así mismo, en las tirotoxicosis, como en la enfermedad de Graves, se han reportado muchos efectos sobre el tejido conectivo de las válvulas tricúspides que conducirían a una degeneración mixomatosa ${ }^{24}$.

\section{Alteración del endocardio}

La tirotoxicosis se asocia con mayor riesgo de arritmias, especialmente supraventriculares. La fibrilación 
auricular es la arritmia más frecuente y se presenta en el $9-22 \%$ de los pacientes con hipertiroidismo. La fibrilación ventricular asociada a tirotoxicosis se explica por dos posibles mecanismos. El primero es el vasoespasmo coronario, en el que la disfunción endotelial y la hiperreactividad de las células del músculo liso vascular que generan espasmo van a ser seguidas de isquemia miocárdica y, posiblemente, de fibrilación ventricular ${ }^{25}$. El segundo mecanismo se asocia con la repolarización temprana. El mecanismo causante de la elevación del punto $\mathrm{J}$ que se presenta en el síndrome de repolarización temprana aún no está claro, pero sí se ha asociado la participación del gradiente de voltaje transmural entre el endocardio y el epicardio en la activación ventricular del potencial de acción ${ }^{25}$. Esto se explica porque los bloqueadores $\beta$ y la estimulación parasimpática mejoran la elevación del punto $\mathrm{J}$ y del segmento ST mediante la atenuación del calcio ionizado, lo cual genera un aumento del gradiente de voltaje entre el endocardio y el epicardio, y de esta forma causan la arritmia ${ }^{26}$. Por ende, el tratamiento indicado para la tormenta tiroidea en pacientes que presentan repolarización temprana podría exacerbar la elevación del punto $\mathrm{J}$ e inducir fibrilación ventricular ${ }^{27}$.

\section{Alteración del miocardio}

En esta estructura se produce un aumento en la contractilidad cardíaca, el gasto cardíaco y la frecuencia cardíaca en reposo. Las funciones sistólica y diastólica mejoran, y la disminución de la resistencia vascular genera una disminución en la poscarga. Estos cambios se ven reflejados en la hemodinámica cardiovascular, lo que conlleva un aumento del flujo sanguíneo y de la perfusión tisular, que se ven reflejados en arritmias auriculares, taquicardia, presión de pulso ensanchada y disnea de esfuerzo ${ }^{6,9}$. El exceso de TSH puede conducir a una disminución en la resistencia vascular periférica y un aumento en el volumen de sangre, lo que resulta en mayores contractilidad y gasto cardíaco. Para compensar este mayor nivel de estrés, se cree que el corazón sufre un proceso de remodelación, caracterizado por el agrandamiento de los miocitos y la deposición de la matriz extracelular. Durante la fase inicial del hipertiroidismo, la compensación se produce por el crecimiento proporcional en el área de corte transversal de los miocitos. Sin embargo, esta carga hemodinámica sostenida puede sobrecargar la capacidad compensatoria del corazón y provocar ventrículos dilatados con mayor longitud de miocitos, sin un aumento adicional en el grosor del diámetro de la pared posterior del ventrículo izquierdo. Esto puede conducir a insuficiencia cardíaca dilatada $^{13,23}$.

Mavrogeni et al. ${ }^{28}$ investigaron la relación entre la enfermedad tiroidea y la miocarditis en 250 pacientes con hipertiroidismo, antitiroglobulina y anticuerpos antimicrosomales elevados, a pesar de que estaban eutiroideos por el tratamiento. De estos, 50 pacientes tenían síntomas cardíacos, como dolor torácico, disnea y palpitaciones. La biopsia endomiocárdica reveló infiltración linfocítica sin infección viral, indicativa de etiología autoinmunitaria ${ }^{28}$. A diferencia de la serie de casos anterior, Lancaster et al. ${ }^{29}$ reportan el caso de un paciente de 29 años con enfermedad de Graves de inicio reciente que presentaba síntomas activos de hipertiroidismo, T4 elevada, TSH suprimida y aparición aguda de síntomas cardíacos con enzimas cardíacas elevadas. El paciente comenzó tratamiento con metimazol, $10 \mathrm{mg}$ dos veces al día, y 3 meses después de su hospitalización experimentó la resolución de sus síntomas cardíacos, con lo que concluyen que esta es una presentación rara concurrente de la enfermedad de Graves y la miocarditis autoinmunitaria ${ }^{29}$. Alam y Zaman ${ }^{30}$ presentan el caso de una paciente de 65 años derivada de atención primaria por empeoramiento de la disnea durante 3 semanas, asociada a taquicardia y bloqueo de rama izquierda. Tenía antecedentes de diabetes mellitus tipo 2, asma e hipertensión. El electrocardiograma inicial reveló fibrilación auricular con frecuencia ventricular rápida. Las pruebas iniciales, incluida la prueba de función tiroidea, revelaron hipertiroidismo. Con esto, se evidenció que la paciente tenía miocardiopatía tirotóxica y se inició tratamiento temprano, con recuperación notable.

\section{Alteración del pericardio}

La enfermedad de Graves es una enfermedad autoinmunitaria en la que la tiroides se activa por anticuerpos contra el receptor de $\mathrm{TSH}^{31}$. Esta enfermedad es conocida por producir alteraciones cardiovasculares, pero son pocos los reportes de pericarditis aguda asociados a esta ${ }^{32}$. Koo et al. ${ }^{32}$ informan sobre un paciente de 42 años con pericarditis recurrente aguda que se consideró asociada con la enfermedad de Graves. En este no se realizó diagnóstico tisular de la pericarditis que revelara la infiltración linfocítica, pero con el curso del paciente, que no presentó recurrencias con el tratamiento de la tiroides, queda la posibilidad de la relación existente entre la inflamación pericárdica 
y la enfermedad de Graves. Mirić et al. ${ }^{33}$ informan el caso de un paciente con perimiocarditis recurrente aguda asociada con tirotoxicosis; al igual que en el caso anterior, este paciente presentó mejoría clínica y normalización de los resultados de laboratorio después del tratamiento de la tirotoxicosis, lo que sugiere el vínculo entre esta y la perimiocarditis recurrente.

\section{Alteración en la circulación coronaria}

Los pacientes que desarrollan hipertiroidismo grave pueden presentar vasoespasmo coronario que se manifiesta con dolor torácico en reposo 0 isquemia miocárdica ${ }^{34}$. En respuesta a la acción de las hormonas tiroideas, el corazón y la vasculatura influyen en la hemodinámica cardiovascular, produciendo un aumento en la contractilidad, pero una disminución en la resistencia vascular, debido a los efectos directos de T3 sobre la termogénesis de los tejidos, la resistencia vascular del sistema y la cronotopía e isotropía cardíacas, lo que resulta en cambios en el volumen sanguíneo y el gasto cardíaco ${ }^{6,9}$.

Según la literatura, los datos ecocardiográficos muestran que el hipertiroidismo a corto plazo induce cambios en el sistema cardiovascular, pues mejora la función sistólica del ventrículo izquierdo y la relajación del mismo. Sin embargo, a medida que evoluciona este cuadro, y a pesar del alto gasto cardíaco, los pacientes con hipertiroidismo presentan una función cardiopulmonar deteriorada durante el esfuerzo, lo que refleja una reserva cardiovascular y respiratoria reducida durante el ejercicio ${ }^{34}$.

\section{Alteración del sistema eléctrico del corazón}

La hormona triyodotironina aumenta la despolarización sistólica y la repolarización diastólica, y disminuye la duración del potencial de acción, así como el período refractario del miocardio auricular y ganglionar auricular/ventricular. Según lo anterior, al generarse un potencial de acción interauricular reducido, se facilita la aparición de fibrilación auricular al mejorar la propagación de la actividad ectópica desde la aurícula izquierda ${ }^{16,34}$.

Asimismo, para el desarrollo de fibrilación auricular en el paciente con hipertiroidismo se debe tener en cuenta el aumento en la edad, la cardiopatía isquémica y la insuficiencia cardíaca congestiva o valvulopatía ${ }^{34}$. Un estudio analizó los efectos de la hormona tiroidea en la actividad arritmogénica de los cardiomiocitos de la vena pulmonar en conejos ${ }^{22}$. Los autores encontraron que la hormona tiroidea incrementaba la actividad espontánea de los cardiomiocitos en la vena pulmonar, y producía una elevación en la aparición de posdespolarizaciones tardías en los cardiomiocitos de dicha vena y un aumento de las despolarizaciones posteriores en los cardiomiocitos vencidos. Con los cambios observados, los autores concluyeron que la hormona tiroidea desempeña un papel en la arritmogénesis $^{22}$. Del $10 \%$ al $25 \%$ de los pacientes con hipertiroidismo tienen fibrilación auricular, y el extremo superior de ese rango lo constituyen los pacientes con hipertiroidismo de 60 años o más; por el contrario, solo el $5 \%$ de los pacientes menores de 60 años con hipertiroidismo tienen fibrilación auricular, 6,16,35.

\section{Conclusiones}

Las enfermedades endocrinas, como el hipertiroidismo y el hipotiroidismo, deben ser identificadas a tiempo, dado que su tratamiento inadecuado induce cambios en el sistema cardiovascular que llevan al desarrollo de cardiopatías que pueden llegar a comprometer la vida del paciente.

\section{Financiamiento}

La presente investigación no ha recibido ninguna beca específica de agencias de los sectores público, comercial o sin ánimo de lucro.

\section{Conflicto de intereses}

Los autores declaran no tener ningún conflicto de intereses.

\section{Responsabilidades éticas}

Protección de personas y animales. Los autores declaran que para esta investigación no se han realizado experimentos en seres humanos ni en animales.

Confidencialidad de los datos. Los autores declaran que en este artículo no aparecen datos de pacientes.

Derecho a la privacidad y consentimiento informado. Los autores declaran que en este artículo no aparecen datos de pacientes.

\section{Bibliografía}

1. Chaves W. Prevalencia de la disfunción tiroidea en la población adulta mayor de la consulta externa del hospital de San José. Acta Médica Colomb. 2018;43:24-30. 
2. Kumar V, Abbas A, Aster J. Robbins y Cotran. Patología estructural y funcional. 9. ${ }^{\mathrm{a}}$ ed. Barcelona: Elsevier; 2014.

3. Soto J, Verbeke S. Disfunción tiroidea y corazón. Rev Médica Clínica Las Condes. 2015;26:186-97.

4. Espinosa A. Mujer, corazón y tiroides. Rev Colomb Cardiol. 2018;25(Supl 1):42-8.

5. Vélez J, Vélez P, Aguayo S, Vélez J, Navarrete R, Tercero W, et al. La glándula tiroides en el paciente críticamente enfermo : abordaje fisiológico y revisión de la literatura. Rev Científica INSPILIP. 2019;3:1-20.

6. Dischinger $U$, Fassnacht $M$. Thyroid gland and the heart: pathophysiological background, diagnostic and therapeutic consequences. Internist. 2018;59:668-73

7. Martínez F. Thyroid hormones and heart failure. Heart Fail Rev 2016;21:361-4.

8. Dan GA. Thyroid hormones and the heart. Heart Fail Rev. 2016;21:357-9.

9. Danzi S, Klein I. Thyroid abnormalities in heart failure. Heart Fail Clin. 2020;16:1-9.

10. Udovcic M, Herrera R, Patham B, Tabatabai L, Kansara A. Hypothyroidism and the heart. Methodist Debakey Cardiovasc J. 2017;13:55-9.

11. Grais IM, Sowers JR. Thyroid and the heart. Am J Med. 2014;127:691-8.

12. Martínez J, Marcos J, Gualis J, Martin C, Martin E, Otero J, et al. Subclinical hypothyroidism might increase the risk of postoperative atrial fibrillation after aortic valve replacement. Thorac Cardiovasc Surg. 2015;64:427-33.

13. Iervasi $G$, Nicolini $G$. Thyroid hormone and cardiovascular system: from basic concepts to clinical application. Intern Emerg Med. 2013;8(Suppl 1):71-4.

14. Klein I, Danzi S. Thyroid disease and the heart. Curr Probl Cardiol. 2016;41:65-92.

15. Jabbar A, Pingitore A, Pearce S, Zaman A, lervasi G, Razvi S. Thyroid hormones and cardiovascular disease. Nat Rev Cardiol. 2017:14:39-55.

16. Vargas $\mathrm{H}$, Bonelo $\mathrm{A}$. Thyroid dysfunction and heart failure: mechanisms and associations. Curr Heart Fail Rep. 2017;14:48-58.

17. Biondi B, Klein I. Hypothyroidism as a risk factor for cardiovascular disease. Endocrine. 2004;24:1-13.

18. Vargas $\mathrm{H}$, Bonelo A, Sierra $\mathrm{C}$. Effects of thyroid hormones on the heart. Clin Invest Arterioscler. 2014;26:296-309.

19. Mirwais S, Kazmi S, Hussain S, Mirwais M, Sharma A. Hypothyroidism causing pericardial effusion: a case report. Cureus. 2019;11:10-4.
20. Delitala A, Fanciulli G, Maioli M, Delitala G. Subclinical hypothyroidism, lipid metabolism and cardiovascular disease. Eur J Intern Med. 2017;38:17-24.

21. Badawy E, Abdel S, Ghonium S. Thyroid stimulating hormone as risk factor for coronary heart disease. J Saudi Heart Assoc. 2013;25:109.

22. Reddy V, Taha W, Kundumadam S, Khan M. Atrial fibrillation and hyperthyroidism: a literature review. Indian Heart J. 2017;69:545-50.

23. Khan R, Sikanderkhel S, Gui J, Adeniyi A, O'Dell K, Erickson M, et al. Thyroid and cardiovascular disease: a focused review on the impact of hyperthyroidism in heart failure. Cardiol Res. 2020;11:68-75.

24. Pierre K, Gadde S, Omar B, Awan GM, Malozzi C. Thyrotoxic valvulopathy: case report and review of the literature. Cardiol Res. 2017:8:134-8.

25. Kobayashi H, Haketa A, Abe M, Tahira K, Hatanaka Y, Tanaka S, et al. Unusual manifestation of Graves' disease: ventricular fibrillation. Eur Thyroid J. 2015:4:207-12.

26. Gussak I, Antzelevitch C. Early repolarization syndrome: clinical characteristics and possible cellular and ionic mechanisms. J Electrocardiol. 2000;33:299-309.

27. Ueno A, Yamamoto T, Sato N, Tanaka K. Ventricular fibrillation associated with early repolarization in a patient with thyroid storm. J Interv Card Electrophysiol. 2010;29:93-6.

28. Mavrogeni S, Markussis V, Bratis K, Mastorakos G, Sidiropoulou E Papadopoulou E, et al. Hyperthyroidism induced autoimmune myocarditis. Evaluation by cardiovascular magnetic resonance and endomyocardial biopsy. Int J Cardiol. 2012;158:166-8.

29. Lancaster S, Koons K, Lee Y, Mazimba S, Kwon Y. Acute autoimmune myocarditis as a manifestation of Graves' disease: a case report and review of the literature. Clin Case Reports. 2019;7:1489-93.

30. Alam S, Zaman J. Case study of thyrotoxic cardiomyopathy. BMJ Case Rep. 2019;12:1-4

31. Smith T, Hegedüs L. Graves' disease. N Engl J Med. 2016;375:1552-65.

32. Koo E, Kim S, Park S, Park J, Kim E, Lee G, et al. Acute recurrent pericarditis accompanied by Graves' disease. Korean Circ J. 2012;42:419-22.

33. Mirić $D$, Glavaš $D$, Kuščić $L$, Kramarić $D$, Lukin $A$, Klančnik $M$, et al. Thyrotoxicosis as the cause of acute recurrent perimyocarditis. Endocr Oncol Metab. 2018:4:2-5.

34. Razvi S, Jabbar A, Pingitore A, Danzi S, Biondi B, Klein I, et al. Thyroid hormones and cardiovascular function and diseases. J Am Coll Cardiol. 2018;71:1781-96.

35. Mejia P, Udovcic M, Sharma M. Hyperthyroidism and the heart. Methodist Debakey Cardiovasc J. 2017;13:60-3. 